\begin{tabular}{|c|c|}
\hline Citation & $\begin{array}{l}\text { Hendrik Mothes, Sandra Klaperski, Harald Seelig, Stefan Schmidt, Reinhard } \\
\text { Fuchs, } 2014 \\
\text { Regular aerobic exercise increases dispositional mindfulness in men: A } \\
\text { randomized controlled trial } \\
\text { Mental Health and Physical Activity, } 7 \text { (2), 111-119. }\end{array}$ \\
\hline Archived version & $\begin{array}{l}\text { Author manuscript: the content is identical to the content of the published } \\
\text { paper, but without the final typesetting by the publisher }\end{array}$ \\
\hline Published version & http://www.sciencedirect.com/science/article/pii/S1755296614000076 \\
\hline Journal homepage & http://www.journals.elsevier.com/mental-health-and-physical-activity/ \\
\hline Author contact & $\begin{array}{l}\text { hendrik.mothes@sport.uni-freiburg.de } \\
+49 \text { (0)761 } 2034563\end{array}$ \\
\hline
\end{tabular}

(article begins on next page) 
REGULAR AEROBIC EXERCISE INCREASES MINDFULNESS

\title{
Regular Aerobic Exercise Increases Dispositional Mindfulness in Men: A Randomized Controlled Trial
}

\author{
Hendrik Mothes ${ }^{\mathrm{a},}$, Sandra Klaperski ${ }^{\mathrm{a}}$, Harald Seelig ${ }^{\mathrm{a}, 1}$, Stefan Schmidt $^{\mathrm{b}, \mathrm{c}}$, Reinhard Fuchs ${ }^{\mathrm{a}}$ \\ ${ }^{\text {a }}$ Institute of Sports Science, University of Freiburg, Schwarzwaldstr. 175, D-79117 Freiburg, Germany; \\ hendrik.mothes@sport.uni-freiburg,de; $\quad$ sandra.klaperski@sport.uni-freiburg.de; $\quad$ harald.seelig@sport.uni- \\ freiburg.de; reinhard.fuchs@sport.uni-freiburg.de \\ ${ }^{\mathrm{b}}$ Department of Psychosomatic Medicine, University Medical Center Freiburg, Hauptstr. 8, 79104 Freiburg, \\ Germany; stefan.schmidt@uniklinik-freiburg.de \\ ${ }^{c}$ Institute of Transcultural Health Studies, European University Viadrina, Frankfurt (Oder), Große Scharrnstr. \\ 59, 15230 Frankfurt (Oder), Germany
}

* Corresponding author:

Hendrik Mothes

Institute of Sports Science, University of Freiburg

Schwarzwaldstraße 175, D-79117 Freiburg, Germany

Phone: +49 761203 4563; fax: +49761 2034534

E-mail address: hendrik.mothes@ sport.uni-freiburg.de

\footnotetext{
${ }^{1}$ Present address: Institute of Sports Science, University of Basel, Birsstrasse 320 B, CH-4052 Basel, Switzerland; harald.seelig@unibas.ch
} 


\title{
REGULAR AEROBIC EXERCISE INCREASES MINDFULNESS
}

\begin{abstract}
Dispositional mindfulness is a construct described as the propensity to be aware of one's actions in everyday life. Although high dispositional mindfulness has been demonstrated to be beneficial for improved mental and physical health, little is known about ways to improve dispositional mindfulness for individuals not practicing meditation or mindful exercises. The study aimed at investigating (1) whether dispositional mindfulness can also be trained by regular aerobic exercise and (2) whether changes in dispositional mindfulness are associated with changes in mental and physical health. 149 healthy men were randomly allocated to one of two 12-week interventions (aerobic exercise or relaxation training) or a waitlist control condition. Dispositional mindfulness and mental and physical health were assessed before and after the intervention by self-report questionnaires. Over the course of the intervention, increases in dispositional mindfulness occurred in the aerobic exercise group but not in the relaxation or waitlist control conditions $(p=.018)$. Increases in dispositional mindfulness were moderately correlated with improvements in mental health. For the first time, this study shows that dispositional mindfulness can be increased through regular aerobic exercise. Future research is needed to identify how the mindfulness-enhancing potential of aerobic exercise can be used most effectively.
\end{abstract}

Keywords: Aerobic exercise, Health behavior, Mindfulness, Mental health, Relaxation, Randomized controlled trial 
REGULAR AEROBIC EXERCISE INCREASES MINDFULNESS

\section{Introduction}

In recent health-related literature, mindfulness has received considerable attention (Baer, 2003). The concept of mindfulness originated in Buddhist tradition and is defined as "paying attention in a particular way: on purpose, in the present moment, and nonjudgmentally" (Kabat-Zinn, 1994, p. 4). A variety of physiological and mental health benefits of mindfulness for clinical and nonclinical populations have been shown in earlier research (Grossman, Niemann, Schmidt, \& Walach, 2004; Hofmann, Sawyer, Witt, \& Oh, 2010; Sedlmeier et al., 2012). Results indicate that mindfulness-based interventions promote health in individuals suffering from cancer, heart disease, chronic pain, fibromyalgia, psoriasis, borderline personality disorder, major depressive disorder, anxiety, and stress.

Besides the notion of mindfulness as a mental state that occurs during or subsequent to mindfulness meditation exercises, mindfulness can also be understood as a dispositional construct that is described as a basic human capacity representing the propensity to act mindfully in everyday life (Brown \& Ryan, 2003; Brown, Ryan, \& Creswell, 2007). This means that a person with high dispositional mindfulness is more often aware of his or her actions and thoughts in contrast to a more dissociative or "auto-pilot" mode. Dispositional mindfulness can be fostered by clinical interventions such as mindfulness-based stress reduction (MBSR) (Carmody \& Baer, 2008; Kabat-Zinn, 1990) as well as by non-clinical mind-body practices such as yoga (Brisbon \& Lowery, 2011), Pilates (Caldwell, Harrison, Adams, Quin, \& Greeson, 2010) or tai chi (Caldwell, Emery, Harrison, \& Greeson, 2011). In turn, such increases in dispositional mindfulness have been shown to lead to better psychological well-being and perceived health (Bränström, Duncan, \& Moskowitz, 2011; Carmody \& Baer, 2008; Murphy, Mermelstein, Edwards, \& Gidycz, 2012).

Recently, movement-based mind-body practices such as yoga or Pilates, referred to as mindful exercises due to their strong focus on body awareness, have become increasingly 


\section{REGULAR AEROBIC EXERCISE INCREASES MINDFULNESS}

popular for fostering mindfulness and health in everyday life. For instance, data from the 2007 National Health Interview Survey indicated that roughly $9 \%$ of the adult population of the United States engages in mindful exercises (Barnes, Bloom, \& Nahin, 2008). Mindful exercises are a new category of physical exercise that are characterized by low to moderate exercise intensities executed with profound and nonjudgmental attention to breathing and proprioceptive awareness of muscles and movements (La Forge, 2005; Larkey, Jahnke, Etnier, \& Gonzalez, 2009). Only recently has it been suggested that mind-body practices incorporating bodily movements are particularly effective. Specifically, Carmody and Baer (2008) found that in MBSR programs, participants benefited more from the typically included movement-based component yoga than from non-movement-based components such as body scan or sitting meditation. The authors argued that it may be easier for individuals to direct mindful attention to their body when engaged in movement. This, in turn, may promote long-lasting mind-body awareness. Further, stressing the importance of movements in enhancing mindfulness, Asztalos and colleagues (2012) recently proposed a theory proclaiming that regular physical exercise performed in a mindful way may contribute to the development of dispositional mindfulness in individuals. They argued that repeated practice of mindful movement is likely to train individuals to experience their own movements, breathing, feelings, and thoughts, while at the same time they will learn to watch these experiences as a bystander.

Aside from mindful exercises, some researchers have raised the question whether involvement in regular physical activity per se (i.e., without explicit instructions to direct attention in a specific way to body and movements, e.g., aerobic exercise) has the potential to increase dispositional mindfulness (e.g., Kee \& Wang, 2008; Salmon, Hanneman, \& Harwood, 2010; Ulmer, Stetson, \& Salmon, 2010). Indeed, aerobic exercises such as swimming, cycling, or jogging share a variety of characteristics with many mindful exercises. 


\section{REGULAR AEROBIC EXERCISE INCREASES MINDFULNESS}

Both types of exercise contain predictive breathing and movement patterns, are repetitive in nature, and are often characterized by an absence of competition. However, they differ from each other, because aerobic exercises are not performed with a deliberate nonjudgmental attention to body and mind (Berger \& Owen, 1988; Netz \& Lidor, 2003). Nevertheless, several researchers have suggested that aerobic exercise affects dispositional mindfulness, potentially through inherent body- and mind-related experiences associated with exercising or via an improved capacity to self-regulate attention. For instance, Kee and Wang (2008) suggested that involvement in physical exercise may lead to increased mindfulness through the opportunities it fosters for moment-to-moment attention. Likewise, another study contended that long-distance running, similarly to meditation practice, promotes "sustained, essentially nonjudgmental attention that can be directed at will toward a wide range of internal and external experiential cues" (Salmon et al., 2010, p. 150). However, compared with the relatively large body of research showing increases in mindfulness following mindful exercises (Brisbon \& Lowery, 2011; Caldwell et al., 2011; Caldwell et al., 2010), there is very little empirical research examining the potentially positive effects of regular aerobic exercise on dispositional mindfulness.

So far, only a few cross-sectional studies have examined the relationship between dispositional mindfulness and regular physical activity (Gilbert \& Waltz, 2010; Murphy et al., 2012; Roberts \& Danoff-Burg, 2010; Ulmer et al., 2010). Only one study investigating 441 women via questionnaires reported no relationship between dispositional mindfulness and regular physical activity (Murphy et al., 2012). In contrast, two studies based on surveys using samples of students showed that higher levels of regular physical activity were moderately associated with higher levels of dispositional mindfulness (Gilbert \& Waltz, 2010; Roberts \& Danoff-Burg, 2010). Similarly, Ulmer and colleagues (2010) reported that YMCA exercisers who were more successful in exercising regularly over the previous year 


\section{REGULAR AEROBIC EXERCISE INCREASES MINDFULNESS}

indicated higher levels of mindfulness. Generally, these results were interpreted as supporting the hypothesis that higher mindfulness leads to improved health behavior (e.g., more physical activity). However, as these studies were cross-sectional in nature, several of these authors conceded that the available data could also be interpreted as being in a reverse causal relationship. In fact, Brown and Ryan (2003)—both initially substantiating the concept of dispositional mindfulness - presumed that there is a bidirectional relationship between mindfulness and health behaviors such as physical activity. To ascertain the causal effects of regular physical activity on dispositional mindfulness, it will be necessary to conduct a randomized controlled trial with regular physical activity as the grouping variable (some receive regular physical activity, others do not) and dispositional mindfulness as the outcome parameter. To the best of our knowledge, a study like this has not been realized so far. The present investigation is an attempt to fill this gap.

In light of the above, the main purpose of this study was to investigate- - by means of a 12-week randomized three-armed intervention - whether initially inactive men participating in a regular aerobic exercise program report greater increases in dispositional mindfulness than the participants of a waitlist control group or relaxation group. Furthermore, we examined on an exploratory level whether resulting changes in dispositional mindfulness were associated with changes in mental and physical health.

\section{Methods}

\section{Study Design}

This study was part of a trial examining psychobiological effects of exercise training on psychosocial stress reactivity (for more details, see Klaperski, von Dawans, Heinrichs, \& Fuchs, 2013). We conducted a randomized, controlled trial utilizing a 12-week intervention period with three groups: (1) exercise training (EG), (2) relaxation training (RG), and (3) waitlist control group (WCG). We hypothesized that (1) participants in the exercise group 


\section{REGULAR AEROBIC EXERCISE INCREASES MINDFULNESS}

would show greater improvements in dispositional mindfulness than those in the nonexercising control groups (RG \& WCG) and (2) development in dispositional mindfulness would not differ between the non-exercising control groups (RG \& WCG). After determining their eligibility (T0), we randomly allocated the participants to one of three study arms following simple randomization procedures (computerized random numbers). The participants were assessed both 1-2 weeks before (T1) and after (T2) the intervention period. Data was collected on two cohorts between February and December 2012. A priori power analyses to compute the required sample size demonstrated that a total sample size of 95 participants was necessary to detect a medium to large effect (as expected due to previous findings; Caldwell et al., 2010) with a power of at least .80 in analysis of covariance.

\section{Participants}

Inclusion criteria for participation were males with predominantly sedentary occupations who reported no or hardly any regular engagement in exercise and relaxation techniques during the previous two months. To recruit participants, we contacted banks, insurance companies, and public services institutions in Freiburg (Germany) and invited all male employees to participate in a study about "Activity and Health.” Exclusion criteria were: (1) severe acute or chronic medical conditions as well as current psychiatric or psychotherapeutic treatment; (2) medical conditions precluding their participation in an exercise or relaxation program; (3) substance abuse, and (4) lack of fluency in German. Participants were not remunerated. However, as a token of gratitude, their exercise or relaxation training was free; additionally, they were offered free running shoes before the intervention, free fitness tests both before and after the intervention, and coupons for fitness and relaxation programs after participation in the study. All participants gave their written informed consent to participate in the experiment, and the procedure was approved by the University of Freiburg Ethics Commission. 


\section{REGULAR AEROBIC EXERCISE INCREASES MINDFULNESS}

The flow of participants throughout the study is presented in Figure 1. A total of 474 individuals completed the eligibility screening (T0) via a questionnaire. Of these, 149 met the inclusion/exclusion criteria and were randomly allocated to the study groups. Of these, 142 completed the first assessment (T1) about four weeks later. Over the course of the intervention, eight participants dropped out, resulting in 134 participants who finished the intervention. Of the remaining 134 participants, 123 completed the second assessment after the intervention (T2). Dropouts during the intervention as well as at the assessments T1/T2 occurred mainly due to health problems or scheduling reasons. Additionally, for statistical analyses, five participants had to be excluded because they did not meet the adherence requirements of the exercise and relaxation interventions (self-reported increase in exercise/relaxation activity and participation in more than $50 \%$ of the supervised training sessions). Thus, 118 participants were eligible for statistical analyses. For further sensitivity analyses, we used a subsample of 101 more inactive participants, as described in the Results section.

\section{Procedure}

Testing procedure. Potential participants were screened for eligibility (T0) via a questionnaire and a telephone interview. After assessing eligibility, we invited the participants to the initial assessment (T1) in our laboratories. The participants were informed that the subject of the study was "Activity and Health"; however, further goals of the study were not disclosed. After completion of the 12-week intervention, we again invited the participants for the second assessment (T2). T1 and T2 assessments took place between 3 and 9 p.m. and consisted of a stress assessment (approximately 1h; not part of this study, see Klaperski et al., 2013), questionnaires (approximately 1h), and thereafter an ergometer fitness test (approximately $1 \mathrm{~h})$. 


\section{REGULAR AEROBIC EXERCISE INCREASES MINDFULNESS}

Intervention. The participants were randomly allocated to one of three groups (see Fig. 1). Subsequent to T1, $n=50$ participants started with the aerobic exercise training (EG), $n=50$ participants started with the relaxation training $(\mathrm{RG})$, and $n=42$ were in the waitlist control group (WCG). The two interventions were comparable in terms of training amounts ( $2 \times 60 \mathrm{~min} /$ week). In both groups, one of the sessions each week was supervised. A second session was performed individually by the participants according to written instructions. The supervised training was conducted by one of two female instructors and usually took place in groups of about five to eight participants at the Institute of Sports Science (University of Freiburg) between 3 and 8 p.m. Adherence to the training sessions was monitored by training journals and heart rate devices (Beurer PM 70).

Exercise training group. The participants executed an outdoor heart rate-controlled running training for beginners. During the first four weeks, the participants alternated between walking and running in order to get used to the training load. After five weeks, the participants ran, if possible, continuously at an intensity of $60-80 \%$ of their maximal heart rate as determined by the lactate test at T1 (Janssen, 2001). The participants were informed about running technique and instructed to warm up before and cool down after running.

Relaxation training group. The participants underwent an indoor relaxation program for beginners consisting mainly of progressive muscle relaxation and autogenic training (Smith, 1999). During relaxation, the participants mostly laid on the ground; no relaxation exercise required any strenuous movements. In the course of the program, the participants were told about relaxation techniques and their principles; mindfulness was not addressed at any time. The RG was chosen as a comparison condition in which participants were engaged in social interactions and had similar training time as in the EG, but without regular aerobic exercise (see Klaperski et al., 2013). 
REGULAR AEROBIC EXERCISE INCREASES MINDFULNESS

Waitlist control group. Members of the WCG received no treatment. After completion of the study they were offered a training program of their choice (aerobic exercise and/or relaxation).

\section{Measures}

Self-attributed dispositional mindfulness. We used the German version of Brown and Ryan's (2003) Mindfulness Attention Awareness Scale (MAAS) to assess self-report dispositional mindfulness in everyday life. This scale is suitable for a general adult population that does not have previous experience with the concept of mindfulness or its specific vocabulary. The MAAS consists of 15 items (e.g., "I find myself doing things without paying attention"), all of which are directed towards mindlessness (absence of mindfulness), as this is more accessible for most individuals than mindfulness (Brown \& Ryan, 2003). The participants were instructed to rate how frequently they experienced each scenario during their everyday life on a 6-point scale ranging from almost always (1) to almost never (6). Both the original English version and the German version (Michalak, Heidenreich, Ströhle, \& Nachtigall, 2008) have a strong one-dimensional factor structure and good psychometric properties (MacKillop \& Anderson, 2007). The internal consistency of the MAAS at T1 and T2 in the present study was $\alpha=.87$ and $\alpha=.90$, respectively.

Exercise and activity measures. To measure exercise and activities of daily living, we used the validated BSA questionnaire (Fuchs, Klaperski, Gerber, \& Seelig, 2013). Physical exercise: The participants named a maximum of three exercise activities they had regularly engaged in during the previous four weeks and indicated the frequency and duration per episode in minutes for each activity. We checked whether each activity they had named was a valid physical exercise activity, considering as valid only exercise activities involving larger groups of skeletal muscles that are intensive enough to lead to significant body experiences. In total, six activities named by the participants (e.g., riding a motor bike, 


\section{REGULAR AEROBIC EXERCISE INCREASES MINDFULNESS}

walking a dog) were classified as invalid and excluded from further calculations. For each valid exercise activity, we computed an index, "physical exercise," in minutes per week. In order to adjust for some less intensive or very long-lasting exercise activities typically containing many resting periods, we considered only half of the time periods for hiking, Nordic walking, downhill and cross-country skiing, couple dancing, horseback riding, and archery. Relaxation activity: As with physical exercise, we assessed relaxation behavior and calculated an index, "relaxation activity," in minutes per week. Activities of daily life: Participants reported how often per week and for how long per episode they had engaged in five given physical activities (walking, cycling to work, other cycling for transportation, strolling, and physically strenuous household activities [e.g., gardening]). Again, we calculated an index, "activities of daily life," in minutes per week (see Klaperski et al., 2013).

Physical fitness measures. To determine physical fitness at T1 and T2, we performed a standardized incremental exercise test starting at $60 \mathrm{~W}$ and increasing by $25 \mathrm{~W}$ every three minutes until volitional exhaustion on a bicycle ergometer (SRM-Ergometer, Schoberer Messtechnik, Jülich, Germany). The participants were instructed to keep the pedal cadence at approximately 90 revolutions per minute. Before the test, the sitting position was adjusted to each individual's comfort. At the end of each stage we took capillary whole blood lactate samples $(20 \mu 1)$ from the earlobe of each participant in order to measure aerobic endurance capacity. We determined blood lactate concentration according to the enzymaticamperometric principle, using Super GL speedy (Mueller Equipment, Dresden, Germany). As an indicator of physical fitness, we detected the Individual Anaerobic Threshold (IAT) relative to body weight (Roecker, Schotte, Niess, Horstmann, \& Dickhuth, 1998; Wasserman, 2012) using the software Ergonizer® (Freiburg, Germany). We instructed the participants to refrain from physical exercise for three days prior to the tests. Bicycle ergometry was given 


\section{REGULAR AEROBIC EXERCISE INCREASES MINDFULNESS}

preference over treadmill ergometry as this imposed fewer demands on motor coordination for the comparatively inactive sample.

Physical and mental health. We used the German version of the SF-12 Health Survey (Bullinger \& Kirchberger, 1998), a validated 12-item self-administered questionnaire that provides information on perceived health status during the previous four weeks. It addresses eight health aspects: physical functioning, physical role limitations, bodily pain, general health, vitality, social functioning, emotional role limitation, and mental health (Gandek et al., 1998; Ware, Kosinski, \& Keller, 1996, Ware, Kosinski, \& Keller, 2002). These eight dimensions can be combined into two scores for physical (physical health component summary - PCS) and mental (mental health component summary - MCS) health. Both PCS and MCS scores are obtained by summation and compared to a norm with a mean score of 50 and a standard deviation of 10 . They range from 0 to 100 , and higher scores reflect better self-reported health. Besides using the two component scores (PCS, MCS) for physical and mental health, we conducted further analyses for subscales of the SF-12 due to the fact that using the two component scores alone (PCS, MCS) has been criticized recently (Güthlin \& Walach, 2007). According to the suggestion of these authors, we employed the subscales (1) physical functioning and (2) physical role functioning to assess perceived physical health status and the subscales (1) vitality, (2) social functioning, (3) emotional role functioning, and (4) mental health to assess perceived mental health status for improved interpretation of the data.

\section{Data Analysis and Statistics}

All analyses were conducted using IBM SPSS Statistics 21.0. Although the sample was randomized, we used a one-way analysis of variance (ANOVA) to check for potential baseline differences between groups possibly caused by the dropouts. Additionally, we performed paired t-tests to investigate within-group differences between $\mathrm{T} 1$ and T2. To 


\section{REGULAR AEROBIC EXERCISE INCREASES MINDFULNESS}

investigate the intervention effect, we calculated analyses of covariance (ANCOVAs), with pre-exercise scores of self-attributed dispositional mindfulness (T1) and age serving as covariates and post-exercise scores (T2) as the dependent measure. Preliminary testing showed that the homogeneity of regression assumption was met for the ANCOVAs. We addressed our two hypotheses with orthogonal planned contrasts, again adjusting for baseline levels of mindfulness (T1) and age. Contrast 1 compared the exercise group with the nonexercising groups (EG \& WCG); contrast 2 compared the non-exercising groups (EG \& WCG). To investigate the expected relationship between dispositional mindfulness and health measures, we performed correlation analyses based on mean group changes between $\mathrm{T} 1$ and $\mathrm{T} 2$ in these variables. For all analyses, the level of significance was set at $p<.05$. In case of violation of homogeneity of variance, we report Brown-Forsythe F for ANOVA results as a more robust measure.

\section{Results}

Table 1 summarizes the sample characteristics at T1 and T2. As may be seen in Table 1, the groups did not differ at baseline (T1) in terms of age, BMI, physical and mental health, activities of daily life, physical exercise, physical fitness, relaxation activity, and dispositional mindfulness. As expected, over the course of the intervention the participants in the exercise group increased their levels of physical exercise (though not significantly, $d=0.20)$ and physical fitness $(d=0.71)$; participants in the relaxation group increased their level of relaxation activity $(d=1.57)$. None of the groups showed changes in physical or mental health over the course of the intervention (see Table 1). Training adherence during the intervention (not shown in Table 1) did not differ between the exercise and relaxation groups with respect to attendance in supervised sessions, $t(79)=-1.10, p=.277$, and total training time, $t(74)=-.60, p=.553$.

\section{Intervention Effect on Mindfulness}




\section{REGULAR AEROBIC EXERCISE INCREASES MINDFULNESS}

ANCOVA analysis revealed that after adjusting for pre-intervention scores (T1) and age, there were significant differences between the post-intervention scores of dispositional mindfulness (T2) in the 3 groups, $F(2,113)=3.12, p=.048, \eta_{p}{ }^{2}=.052$. In order to address the two hypotheses, we performed two orthogonal planned contrasts: (1) EG vs. RG/WCG and (2) RG vs. WCG, both adjusted for baseline levels of mindfulness and age. The first contrast revealed that the exercise group had higher levels of mindfulness after the intervention compared to the non-exercising control conditions relaxation group and waitlist control group, $t=2.39, p=.018, \eta^{2}=0.05$. The second contrast demonstrated that mindfulness levels after the intervention did not differ in the relaxation group compared to the waitlist control group, $t=-0.64, p=.526, \eta^{2}=0.00$. Analyses on group level using paired t-tests revealed that over the course of the intervention, levels of mindfulness increased with a trend towards significance in the exercise group, $t(39)=-1.77, p=.084, d=.28$, but not in the relaxation group or waitlist control group (both $p \geq .226$ ).

We conducted further sensitivity analyses with a more inactive (defined as less than 60 minutes of physical exercise per week at T1) subsample of 101 participants, because some of the participants that were classified as physically inactive during screening (T0) reported already considerable amounts of exercise before the intervention at T1 (see Figures 1 and 2). The results of a one-way ANCOVA comparing the post-intervention scores of dispositional mindfulness (T2) while controlling for the pre-intervention scores (T1) and age showed that there were significant differences between the 3 groups, $F(2,96)=4.38, p=.015, \eta_{p}{ }^{2}=.084$ (see Figure 2). On group level, paired t-tests showed that over the course of the intervention levels of mindfulness increased in the exercise group, $t(31)=-2.45, p=.020, d=.43$, but not in the relaxation group or waitlist control group (both $p \geq .312$ ). These results support the findings described above and suggest that the effect of physical exercise on dispositional 


\section{REGULAR AEROBIC EXERCISE INCREASES MINDFULNESS}

mindfulness is more pronounced when individuals are physically inactive at the beginning of the intervention.

Taken together, the findings indicate that dispositional mindfulness was increased solely by participation in a regular exercise program (see Figure 2). Neither of the other groups showed enhancements in mindfulness levels over the course of the intervention. Participation in a relaxation program did not affect dispositional mindfulness differently from the waitlist control condition.

\section{Correlations between Dispositional Mindfulness and Health Measures}

Changes in mindfulness and changes in mental health (MCS) were significantly correlated, $r(115)=.199, p=.032$. However, changes in mindfulness were unrelated to changes in physical health (PCS), $r(115)=-.023, p=.806$. Regarding mental health, further analyses using subscales showed that changes in mindfulness were significantly correlated with changes in the subscale emotional role functioning, $r(116)=.188, p=.042$, and correlated by trend with changes in the subscale social functioning, $r(116)=.169, p=.068$. Changes in both other mental health subscales were unrelated to mindfulness (both $p \geq .161$ ). Regarding physical health, the analyses using subscales supported the results employing the component score PCS by showing that increasing mindfulness was correlated neither with changes in physical functioning, $r(116)=.150, p=.106$, nor with changes in physical role functioning, $r(115)=.110, p=.238$.

\section{Discussion}

The present study examined whether participation in a regular aerobic exercise program can increase dispositional mindfulness and whether changes in dispositional mindfulness are associated with changes in measures of mental and physical health. With regard to the first research question, results of the randomized controlled trial showed that dispositional mindfulness increased significantly over the course of the 12-week intervention 


\section{REGULAR AEROBIC EXERCISE INCREASES MINDFULNESS}

in the exercise group, whereas dispositional mindfulness did not change in the relaxation and waitlist control group (Figure 2). To our knowledge, this is the first randomized controlled trial demonstrating that dispositional mindfulness can be favorably affected by regular aerobic exercise. It is important to note that the aerobic exercises included in the intervention were not per se mindful in character (like yoga, Pilates, tai-chi); instead, the aerobic exercise intervention consisted of the everyday sport activity running. Regarding the second research question, we demonstrated on an exploratory level that changes in mindfulness over the period of the intervention were positively associated with changes in mental health but not in physical health. Taken together, these findings shed initial light on the complex interplay between physical exercise and dispositional mindfulness in the process of health promotion. Below, we look at the major results in more detail.

\section{Effects of Physical Exercise on Mindfulness}

Adding to previous research (Gilbert \& Waltz, 2010; Roberts \& Danoff-Burg, 2010; Ulmer et al., 2010), our findings suggest a causal effect of physical activity on mindfulness. But how can this effect by explained? What are the potential mechanisms behind the relationship between physical activity and mindfulness? A first possible explanation refers to improved body awareness through regular aerobic exercise, as the enhancement of body awareness has also been suggested to be a core mode of action of many mindful exercises in increasing mindfulness (for a review: Mehling et al., 2009). Research has suggested that repeated exposure to bodily functions such as increased breathing, heart rate, temperature regulation, and hormone release may enhance body awareness. Specifically, Skrinar and colleagues (1986) showed that previously inactive women increased their body awareness through participation in a 6 to 8 week intensive endurance running program. Therefore, one can speculate that the participants in our sample — initially inactive men-increased their 


\section{REGULAR AEROBIC EXERCISE INCREASES MINDFULNESS}

body awareness by being repeatedly exposed to intensive body experiences. This, in turn, may have led to higher dispositional mindfulness.

Increased dispositional mindfulness following regular aerobic exercise may also be explained by improved self-regulation of attention similar to the effects of mindfulness training. In fact, a primary mechanism of mindfulness training is practicing the ability to sustain attention to the present moment and to switch attention back to the present moment whenever the mind goes astray (Bishop et al., 2004). Similar to mindfulness training, regular and acute aerobic exercise have also been shown to affect attentional performance in recent research (Barnes, Coombes, Armstrong, Higgins, \& Janelle, 2010; Hillman, Erickson, \& Kramer, 2008; Smith et al., 2010). For instance, numerous prospective studies have demonstrated that exercise interventions with a duration of 2 to 12 weeks effectively enhance attentional processes, particularly the ability to sustain attention and to stay cognitively flexible (Ratey \& Loehr, 2011). With respect to self-regulation, a study by Oaten and Cheng (2006) has shown that regular physical exercise led to improvements in a wide range of regulatory behaviors. In addition, neuroscience also provides support for an attention-related explanation, as both training forms substantially affect the functioning of the anterior cingulate cortex, a brain region implicated with improved self-regulation of attention (Colcombe et al., 2004; Hillman et al., 2008; Tang \& Posner, 2009). Moreover, recent research has shown that both aerobic exercise and meditation are accompanied by a transient prefrontal deregulation (Dietrich, 2006; Manna et al., 2010), which is associated with a change in focused attention (Dietrich, 2003). Hence, one may hypothesize that the intervention effect on dispositional mindfulness is the result of improved self-regulation of attention.

\section{Effects of Relaxation on Mindfulness}




\section{REGULAR AEROBIC EXERCISE INCREASES MINDFULNESS}

Our results also indicated that the relaxation intervention did not improve dispositional mindfulness. It is still unknown whether regular practice of relaxation leads to increases in mindfulness (Agee, Danoff-Burg, \& Grant, 2009; Carmody \& Baer, 2008). An explanation for our findings may be that relaxation training does not improve mindfulnessrelevant attention to the same extent that aerobic exercise training does. For instance, two randomized controlled trials revealed that progressive muscle relaxation training did not affect attentional processes when compared to mindfulness training (Semple, 2010; Tang et al., 2007). Furthermore, it is possible that the relaxation training did not appeal to our male sample as much as the aerobic exercise training. Indeed, earlier research has shown that men are less attracted to relaxation practices (Barnes et al., 2008). As a consequence, they may not have benefited as much with respect to relaxation and mindfulness. Such reasoning is in line with previous research indicating that physical exercise has the highest potential for enhancing mental health when personal preferences are accounted for (Asztalos et al., 2012; Berger \& Owen, 1992; Miller, Bartholomew, \& Springer, 2005). However, as we have not directly measured the effectiveness of the relaxation training, this issue needs to be resolved in future research.

\section{Association between Dispositional Mindfulness and Health Status}

As noted above, across groups changes in self-reported dispositional mindfulness (T1 to $\mathrm{T} 2$ ) were positively associated with changes in mental health (T1 to $\mathrm{T} 2$ ), particularly social functioning and emotional role functioning. These findings are consistent with previous research (Keng, Smoski, \& Robins, 2011) and underscore the potential importance of dispositional mindfulness in the promotion of mental health. Contrary to earlier research (Murphy et al., 2012), we found that changes in dispositional mindfulness were unrelated to changes in physical health. One may speculate that the increases in dispositional mindfulness over the 12-week intervention period were not large enough to be accompanied by changes in 


\section{REGULAR AEROBIC EXERCISE INCREASES MINDFULNESS}

physical health. However, as these analyses were exploratory, more rigorous research using mediation analysis is needed in the future.

\section{Limitations and Strengths}

Several limitations of this study should be noted. First, despite the screening for physically inactive participants, at baseline (T1) our sample included 17 participants reporting considerable regular exercise levels of 60 minutes and above. The presence of these rather active participants in our sample explains why the change in physical exercise over the course of the intervention did not reach significance in the exercise group (Table 1). Since a test of our hypothesis required a sample that had been inactive previous to the intervention, we excluded these participants for additional sensitivity analyses. Because a subsample of the randomized sample was used in these analyses, the respective results may be biased by a regression to the mean effect. Second, the sample of healthy men limits the generalizability of these results. Moreover, as we did not directly measure body experience, body awareness, or attentional processes, this study does not allow conclusions about the proposed underlying mechanisms of the effect of aerobic exercise on dispositional mindfulness. In addition, the study is also limited by the fact that we used participants who were naive to the concept of mindfulness. Self-report measurement of mindfulness among such individuals has been criticized in earlier research due to potential semantic misinterpretations of mindfulness items (Grossman, 2008), and this has recently been confirmed empirically for one of the often used instruments (Belzer et al., 2013). We tried to solve this problem by using the MAAS, an economical, reliable, and valid measure that is also adequate for use by participants without experience in the concept of mindfulness (Brown \& Ryan, 2003). On the other hand, the MAAS addresses only one aspect of the mindfulness concept, i.e., habitual present-moment awareness. Other instruments include other important aspects, such as acceptance, non- 


\section{REGULAR AEROBIC EXERCISE INCREASES MINDFULNESS}

reactivity, describing, or observing, which have not been touched upon here. Future studies may also benefit from employing objective measures of mindfulness.

In spite of the limitations, our study has several strengths. For the first time, we showed in a randomized controlled design that regular aerobic exercise can be used to increase dispositional mindfulness in men. This finding is important, since it further advances our understanding of how the health-relevant resource of dispositional mindfulness can be developed outside the context of meditation and mind-body exercises. Prospective mindfulness training through aerobic exercise may have particular relevance for adult men as previous research suggests that they are generally more attracted by aerobic exercises than by mindful exercises. Several publications in popular media (e.g., Mipham, 2012) and research articles (Asztalos et al., 2012) have claimed that physical exercise can be used to increase mindfulness abilities when performed in a mindful way. In light of our results, we claim that this understanding is too narrow and instead propose that even unmindful, regular practice of certain forms of aerobic exercise can lead to increases in dispositional mindfulness. Future research should substantiate this finding and investigate more closely the underlying mechanisms and conditions under which the mindfulness-enhancing potential of aerobic exercise can be used most effectively (e.g., type of exercise, exercise intensity and duration, individual characteristics of the person involved in the exercise) and the practical relevance of such an effect. A likely future study could investigate the difference of changes in dispositional mindfulness between exercise groups with and without explicit mindfulnessrelated instructions. Ultimately, such a systematic use of aerobic exercise will provide a simple and cost-effective opportunity to make better use of the health-relevant resource of mindfulness for a wide range of people.

\section{Conflict of Interest}

The authors declare that they have no conflicts of interest. 
REGULAR AEROBIC EXERCISE INCREASES MINDFULNESS

\section{Acknowledgments}

We thank all those at the Institute of Sports Science at the University of Freiburg who provided support for this study, with special thanks to Julia Jäger for her assistance on this project. The authors also wish to thank Mathis Trautwein, Johannes Sperling, Joshua Marcus, and Jillian DeMair for their comments on this manuscript. This research was funded by the German Research Foundation (research grant FU458/2-1) and the Konrad Adenauer Foundation. 


\section{References}

Agee, J. D., Danoff-Burg, S., \& Grant, C. A. (2009). Comparing brief stress management courses in a community sample: Mindfulness skills and progressive muscle relaxation. EXPLORE: The Journal of Science and Healing, 5(2), 104-109. doi:10.1016/j.explore.2008.12.004

Asztalos, M., Wijndaele, K., Bourdeaudhuij, I. de, Philippaerts, R., Matton, L., Duvigneaud, N., et al. (2012). Sport participation and stress among women and men. Psychology of Sport and Exercise, 13(4), 466-483. doi:10.1016/j.psychsport.2012.01.003

Baer, R. A. (2003). Mindfulness training as a clinical intervention: A conceptual and empirical review. Clinical Psychology: Science and Practice, 10(2), 125-143. doi:10.1093/clipsy/bpg015

Barnes, P. M., Bloom, B., \& Nahin, R. L. (2008). Complementary and alternative medicine use among adults and children: United States, 2007. National Health Statistics Reports, (12), 1-23.

Barnes, R. T., Coombes, S. A., Armstrong, N. B., Higgins, T. J., \& Janelle, C. M. (2010). Evaluating attentional and affective changes following an acute exercise bout using a modified dot-probe protocol. Journal of Sports Sciences, 28(10), 1065-1076. doi:10.1080/02640414.2010.489196

Belzer, F., Schmidt, S., Lucius-Hoene, G., Schneider, J. F., Orellana-Rios, C. L., \& Sauer, S. (2013). Challenging the construct validity of mindfulness assessment — a cognitive interview study of the Freiburg Mindfulness Inventory. Mindfulness, 4(1), 33-44. doi:10.1007/s12671-012-0165-7

Berger, B. G., \& Owen, D. R. (1988). Stress reduction and mood enhancement in four exercise modes: Swimming, body conditioning, hatha yoga, and fencing. Research Quarterly for Exercise and Sport, 59(2), 148-159. doi:10.1080/02701367.1988.10605493 


\section{REGULAR AEROBIC EXERCISE INCREASES MINDFULNESS}

Berger, B. G., \& Owen, D. R. (1992). Mood alteration with yoga and swimming-Aerobic exercise may not be necessary. Perceptual and Motor Skills, 75(3f), 1331-1343. doi:10.2466/pms.1992.75.3f.1331

Bishop, S. R., Lau, M., Shapiro, S., Carlson, L., Anderson, N. D., Carmody, J., et al. (2004). Mindfulness: A proposed operational definition. Clinical Psychology: Science and Practice, 11(3), 230-241. doi:10.1093/clipsy.bph077

Bränström, R., Duncan, L. G., \& Moskowitz, J. T. (2011). The association between dispositional mindfulness, psychological well-being, and perceived health in a Swedish population-based sample. British Journal of Health Psychology, 16(2), 300-316. doi:10.1348/135910710X501683

Brisbon, N. M., \& Lowery, G. A. (2011). Mindfulness and levels of stress: A comparison of beginner and advanced hatha yoga practitioners. Journal of Religion and Health, 50(4), 931-941. doi:10.1007/s 10943-009-9305-3

Brown, K. W., \& Ryan, R. M. (2003). The benefits of being present: Mindfulness and its role in psychological well-being. Journal of Personality and Social Psychology, 84(4), 822848. doi:10.1037/0022-3514.84.4.822

Brown, K. W., Ryan, R. M., \& Creswell, J. D. (2007). Mindfulness: Theoretical foundations and evidence for its salutary effects. Psychological Inquiry, 18(4), 211-237. doi:10.1080/10478400701598298

Bullinger, M., \& Kirchberger, I. (1998). Der SF-36 Fragebogen zum Gesundheitszustand. Handbuch für die deutschsprachige Fragebogenversion [The SF-36 health survey. Manual for the German version of the questionnaire]. Göttingen: Hogrefe.

Caldwell, K., Emery, L., Harrison, M., \& Greeson, J. (2011). Changes in mindfulness, wellbeing, and sleep quality in college students through taijiquan courses: A cohort control 


\section{REGULAR AEROBIC EXERCISE INCREASES MINDFULNESS}

study. The Journal of Alternative and Complementary Medicine, 17(10), 931-938. doi:10.1089/acm.2010.0645

Caldwell, K., Harrison, M., Adams, M., Quin, R. H., \& Greeson, J. (2010). Developing mindfulness in college students through movement-based courses: Effects on selfregulatory self-efficacy, mood, stress, and sleep quality. Journal of American College Health, 58(5), 433-442. doi:10.1080/07448480903540481

Carmody, J., \& Baer, R. A. (2008). Relationships between mindfulness practice and levels of mindfulness, medical and psychological symptoms and well-being in a mindfulness-based stress reduction program. Journal of Behavioral Medicine, 31(1), 23-33. doi:10.1007/s10865-007-9130-7

Colcombe, S. J., Kramer, A. F., Erickson, K. I., Scalf, P. E., McAuley, E., Cohen, N. J., et al. (2004). Cardiovascular fitness, cortical plasticity, and aging. Proceedings of the National Academy of Sciences, 101(9), 3316-3321. doi:10.1073/pnas.0400266101

Dietrich, A. (2003). Functional neuroanatomy of altered states of consciousness: The transient hypofrontality hypothesis. Consciousness and Cognition, 12(2), 231-256. doi:10.1016/S1053-8100(02)00046-6

Dietrich, A. (2006). Transient hypofrontality as a mechanism for the psychological effects of exercise. Psychiatry Research, 145(1), 79-83. doi:10.1016/j.psychres.2005.07.033

Fuchs, R., Klaperski, S., Gerber, M., \& Seelig, H. (2013). Messung der Bewegungs- und Sportaktivität: Der BSA-Fragebogen [Measurement of physical activity, exercise and sport: The BSA questionnaire]. Manuscript submitted for publication.

Gandek, B., Ware, J., Aaronson, N. K., Apolone, G., Bjorner, J. B., Brazier, J. E., et al. (1998). Cross-validation of item selection and scoring for the SF-12 health survey in nine countries. Journal of Clinical Epidemiology, 51(11), 1171-1178. doi:10.1016/S08954356(98)00109-7 


\section{REGULAR AEROBIC EXERCISE INCREASES MINDFULNESS}

Gilbert, D., \& Waltz, J. (2010). Mindfulness and health behaviors. Mindfulness, 1(4), 227234. doi:10.1007/s12671-010-0032-3

Grossman, P. (2008). On measuring mindfulness in psychosomatic and psychological research. Journal of Psychosomatic Research, 64(4), 405-408. doi:10.1016/j.jpsychores.2008.02.001

Grossman, P., Niemann, L., Schmidt, S., \& Walach, H. (2004). Mindfulness-based stress reduction and health benefits: A meta-analysis. Journal of Psychosomatic Research, 57(1), 35-43. doi:10.1016/S0022-3999(03)00573-7

Güthlin, C., \& Walach, H. (2007). MOS-SF 36: Structural equation modeling to test the construct validity of the second-order factor structure. European Journal of Psychological Assessment, 23(1), 15-23. doi:10.1027/1015-5759.23.1.15

Hillman, C. H., Erickson, K. I., \& Kramer, A. F. (2008). Be smart, exercise your heart: Exercise effects on brain and cognition. Nature Reviews Neuroscience, 9(1), 58-65. doi:10.1038/nrn2298

Hofmann, S. G., Sawyer, A. T., Witt, A. A., \& Oh, D. (2010). The effect of mindfulnessbased therapy on anxiety and depression: A meta-analytic review. Journal of Consulting and Clinical Psychology, 78(2), 169-183. doi:10.1037/a0018555

Janssen, P. G. J. M. (2001). Lactate threshold training. Champaign, IL: Human Kinetics. Kabat-Zinn, J. (1990). Full catastrophe living: Using the wisdom of your body and mind to face stress, pain, and illness. New York: Dell.

Kabat-Zinn, J. (1994). Wherever you go, there you are: Mindfulness meditation in everyday life (1st ed.). New York: Hyperion. 


\section{REGULAR AEROBIC EXERCISE INCREASES MINDFULNESS}

Kee, Y. H., \& Wang, J. C. (2008). Relationships between mindfulness, flow dispositions and mental skills adoption: A cluster analytic approach. Psychology of Sport and Exercise, 9(4), 393-411. doi:10.1016/j.psychsport.2007.07.001

Keng, S.-L., Smoski, M. J., \& Robins, C. J. (2011). Effects of mindfulness on psychological health: A review of empirical studies. Clinical Psychology Review, 31(6), 1041-1056. doi:10.1016/j.cpr.2011.04.006

Klaperski, S., von Dawans, B., Heinrichs, M., \& Fuchs, R. (2013). Effects of a 12-week endurance training on the physiological response to psychosocial stress in men: A randomized controlled trial. Manuscript submitted for publication.

La Forge, R. (2005). Aligning mind and body. ACSM's Health \& Fitness Journal, 9(5), 7-14. doi:10.1097/00135124-200509000-00006

Larkey, L., Jahnke, R., Etnier, J., \& Gonzalez, J. (2009). Meditative movement as a category of exercise: Implications for research. Journal of Physical Activity \& Health, 6(2), 230 238.

MacKillop, J., \& Anderson, E. J. (2007). Further psychometric validation of the mindful attention awareness scale (MAAS). Journal of Psychopathology and Behavioral Assessment, 29(4), 289-293. doi:10.1007/s10862-007-9045-1

Mehling, W. E., Gopisetty, V., Daubenmier, J., Price, C. J., Hecht, F. M., Stewart, A., \& García, A. V. (2009). Body awareness: Construct and self-report measures. PLoS ONE, 4(5), e5614. doi:10.1371/journal.pone.0005614

Michalak, J., Heidenreich, T., Ströhle, G., \& Nachtigall, C. (2008). Die deutsche Version der Mindful Attention and Awareness Scale (MAAS) Psychometrische Befunde zu einem Achtsamkeitsfragebogen [German version of the Mindful Attention and Awareness Scale (MAAS) - Psychometric features of a mindfulness questionnaire]. Zeitschrift für Klinische Psychologie und Psychotherapie, 37(3), 200-208. doi:10.1026/1616-3443.37.3.200 


\section{REGULAR AEROBIC EXERCISE INCREASES MINDFULNESS}

Miller, B. M., Bartholomew, J. B., \& Springer, B. A. (2005). Post-exercise affect: The effect of mode preference. Journal of Applied Sport Psychology, 17(4), 263-272. doi:10.1080/10413200500313503

Mipham, S. (2012). Running with the mind of meditation: Lessons for training body and mind. New York: Harmony Books.

Murphy, M. J., Mermelstein, L. C., Edwards, K. M., \& Gidycz, C. A. (2012). The benefits of dispositional mindfulness in physical health: A longitudinal study of female college students. Journal of American College Health, 60(5), 341-348.

doi:10.1080/07448481.2011.629260

Netz, Y., \& Lidor, R. (2003). Mood alterations in mindful versus aerobic exercise modes. The Journal of Psychology, 137(5), 405-419.

Oaten, M., \& Cheng, K. (2006). Longitudinal gains in self-regulation from regular physical exercise. British Journal of Health Psychology, 11(4), 717-733.

doi:10.1348/135910706X96481

Ratey, J. J., \& Loehr, J. E. (2011). The positive impact of physical activity on cognition during adulthood: A review of underlying mechanisms, evidence and recommendations. Reviews in the Neurosciences, 22(2), 171-185. doi:10.1515/RNS.2011.017

Roberts, K. C., \& Danoff-Burg, S. (2010). Mindfulness and health behaviors: Is paying attention good for you? Journal of American College Health, 59(3), 165-173. doi:10.1080/07448481.2010.484452

Roecker, K., Schotte, O., Niess, A. M., Horstmann, T., \& Dickhuth, H. H. (1998). Predicting competition performance in long-distance running by means of a treadmill test. Medicine and Science in Sports and Exercise, 30(10), 1552-1557. 


\section{REGULAR AEROBIC EXERCISE INCREASES MINDFULNESS}

Salmon, P., Hanneman, S., \& Harwood, B. (2010). Associative/dissociative cognitive strategies in sustained physical activity: Literature review and proposal for a mindfulnessbased conceptual model. The Sport Psychologist, (24), 127-156.

Sedlmeier, P., Eberth, J., Schwarz, M., Zimmermann, D., Haarig, F., Jaeger, S., \& Kunze, S. (2012). The psychological effects of meditation: A meta-analysis. Psychological Bulletin, 138(6), 1139-1171. doi:10.1037/a0028168

Semple, R. J. (2010). Does mindfulness meditation enhance attention? A randomized controlled trial. Mindfulness, 1(2), 121-130. doi:10.1007/s12671-010-0017-2

Skrinar, G. S., Bullen, B. A., Cheek, J. M., McArthur, J. W., \& Vaughan, L. K. (1986). Effects of endurance training on body-consciousness in women. Perceptual and Motor Skills, 62(2), 483-490.

Smith, J. C. (1999). ABC relaxation training: A practical guide for health professionals. New York: Springer.

Smith, P. J., Blumenthal, J. A., Hoffman, B. M., Cooper, H., Strauman, T. A., WelshBohmer, K., et al. (2010). Aerobic exercise and neurocognitive performance: A metaanalytic review of randomized controlled trials. Psychosomatic Medicine, 72(3), 239-252. doi:10.1097/PSY.0b013e3181d14633

Tang, Y.-Y., Ma, Y., Wang, J., Fan, Y., Feng, S., Lu, Q., et al. (2007). Short-term meditation training improves attention and self-regulation. Proceedings of the National Academy of Sciences, 104(43), 17152-17156. doi:10.1073/pnas.0707678104

Tang, Y.-Y., \& Posner, M. I. (2009). Attention training and attention state training. Trends in Cognitive Sciences, 13(5), 222-227. doi:10.1016/j.tics.2009.01.009 


\section{REGULAR AEROBIC EXERCISE INCREASES MINDFULNESS}

Ulmer, C. S., Stetson, B. A., \& Salmon, P. G. (2010). Mindfulness and acceptance are associated with exercise maintenance in YMCA exercisers. Behaviour Research and Therapy, 48(8), 805-809. doi:10.1016/j.brat.2010.04.009

Ware, J., Kosinski, M., \& Keller, S. D. (1996). A 12-item short-form health survey. Medical Care, 34(3), 220-233. doi:10.1097/00005650-199603000-00003

Ware, J., Kosinski, M., \& Keller, S. D. (2002). SF-12: How to score the SF-12 physical and mental health summary scales (4th ed.). Lincoln, R.I, Boston, Mass: QualityMetric Inc.; Health Assessment Lab.

Wasserman, K. (2012). Principles of exercise testing and interpretation: Including pathophysiology and clinical applications (5th ed.). Philadelphia: Wolters Kluwer Health/Lippincott Williams \& Wilkins. 
Table 1

Descriptive statistics (mean, SD) of the study groups at T1 and T2 and within-group effect sizes (Cohen's d)

\begin{tabular}{|c|c|c|c|c|c|c|c|c|}
\hline Variables & \multicolumn{3}{|c|}{$\mathrm{EG}(\mathrm{n}=40)$} & \multicolumn{2}{|l|}{ WCG $(\mathrm{n}=37)$} & \multicolumn{2}{|l|}{$\mathrm{RG}(\mathrm{n}=41)$} & $p$ \\
\hline Age (years) & $\mathrm{T} 1$ & $44.45 \pm 9.78$ & & $46.92 \pm 11.93$ & & $44.24 \pm 9.55$ & & .462 \\
\hline Physical health (SF 12a $)$ & $\begin{array}{l}\mathrm{T} 1 \\
\mathrm{~T} 2\end{array}$ & $\begin{array}{l}52.99 \pm 5.21 \\
53.00 \pm 5.21\end{array}$ & 0.03 & $\begin{array}{l}52.98 \pm 4.35 \\
51.80 \pm 4.78\end{array}$ & 0.19 & $\begin{array}{l}51.91 \pm 5.27 \\
50.82 \pm 7.45\end{array}$ & 0.18 & $\begin{array}{l}.534 \\
.267\end{array}$ \\
\hline $\begin{array}{l}\text { Physical exercise } \\
\text { (min/week) }\end{array}$ & $\begin{array}{l}\mathrm{T} 1 \\
\mathrm{~T} 2\end{array}$ & $\begin{aligned} 60.16 & \pm 204.10 \\
101.56 & \pm 50.02\end{aligned}$ & 0.20 & $\begin{array}{l}45.57 \pm 109.29 \\
32.23 \pm 61.13\end{array}$ & 0.13 & $\begin{array}{l}14.73 \pm 50.08 \\
30.30 \pm 56.68\end{array}$ & 0.21 & $\begin{array}{l}.318 \\
.000\end{array}$ \\
\hline $\begin{array}{l}\text { Activities of daily life } \\
\text { (min/week) }\end{array}$ & $\begin{array}{l}\mathrm{T} 1 \\
\mathrm{~T} 2\end{array}$ & $\begin{array}{l}357.88 \pm 253.56 \\
341.79 \pm 242.36\end{array}$ & 0.10 & $\begin{array}{l}289.53 \pm 162.27 \\
302.65 \pm 168.29\end{array}$ & 0.10 & $\begin{array}{l}333.89 \pm 217.64 \\
351.89 \pm 177.80\end{array}$ & 0.09 & $\begin{array}{l}.370 \\
.523\end{array}$ \\
\hline $\begin{array}{l}\text { Physical fitness } \\
\text { (p(IAS)/kg) }\end{array}$ & $\begin{array}{l}\mathrm{T} 1 \\
\mathrm{~T} 2\end{array}$ & $\begin{array}{r}1.65 \pm 0.35 \\
* * * 1.78 \pm 0.33\end{array}$ & 0.71 & $\begin{array}{l}1.61 \pm 0.42 \\
1.65 \pm 0.51\end{array}$ & 0.20 & $\begin{array}{l}1.63 \pm 0.45 \\
1.70 \pm 0.45\end{array}$ & 0.24 & $\begin{array}{l}.922 \\
.474\end{array}$ \\
\hline $\begin{array}{l}\text { Dispositional mindfulness } \\
\text { (MAAS }^{\text {b }} \text { ) }\end{array}$ & $\begin{array}{l}\mathrm{T} 1 \\
\mathrm{~T} 2\end{array}$ & $\begin{array}{r}4.45 \pm 0.62 \\
4.57 \pm 0.73\end{array}$ & 0.28 & $\begin{array}{l}4.51 \pm 0.69 \\
4.49 \pm 0.69\end{array}$ & 0.05 & $\begin{array}{l}4.34 \pm 0.59 \\
4.27 \pm 0.69\end{array}$ & 0.19 & $\begin{array}{l}.501 \\
.138\end{array}$ \\
\hline
\end{tabular}

Note. Significant differences and trend differences among groups are highlighted in boldface; significant differences and trend differences within groups are additionally indicated using asterisks. $d=$ Cohen's d; EG = exercise group; $\mathrm{RG}=$ relaxation group; WCG $=$ waitlist control group.

${ }^{\mathrm{a}}$ SF $12=$ SF-12 Health Survey questionnaire. ${ }^{\mathrm{b}}$ MAAS $=$ Mindfulness Attention Awareness Scale.

${ }^{\dagger} p<.10 . * p<.05 . * * p<.01 . * * *=p<.001$. 


\section{REGULAR AEROBIC EXERCISE INCREASES MINDFULNESS}

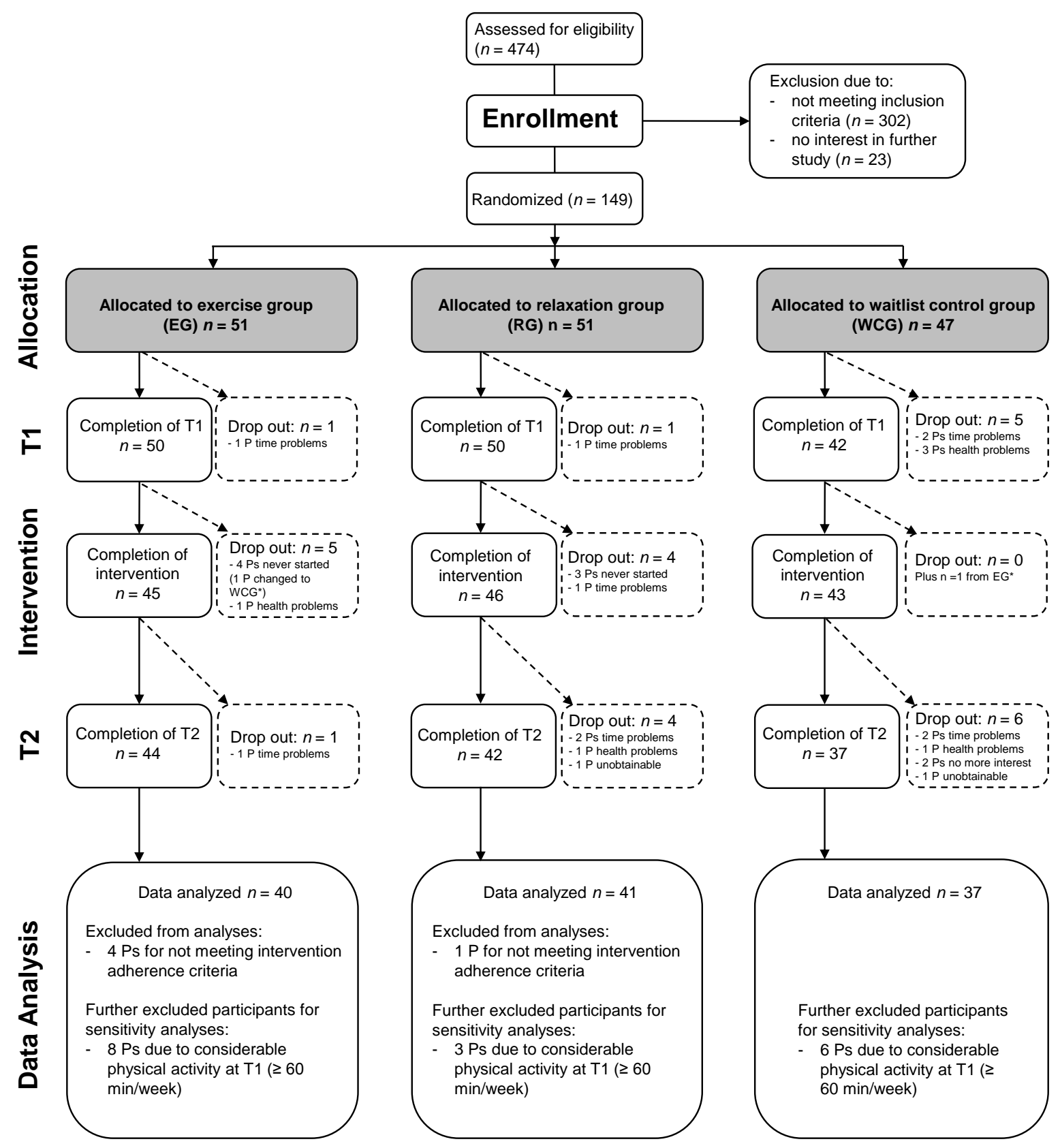

Figure 1. Participant flow chart. $\mathrm{P}(\mathrm{s})=$ participant(s); * $=$ one participant was sick during the first four weeks of the intervention and switched to the waitlist control group. 


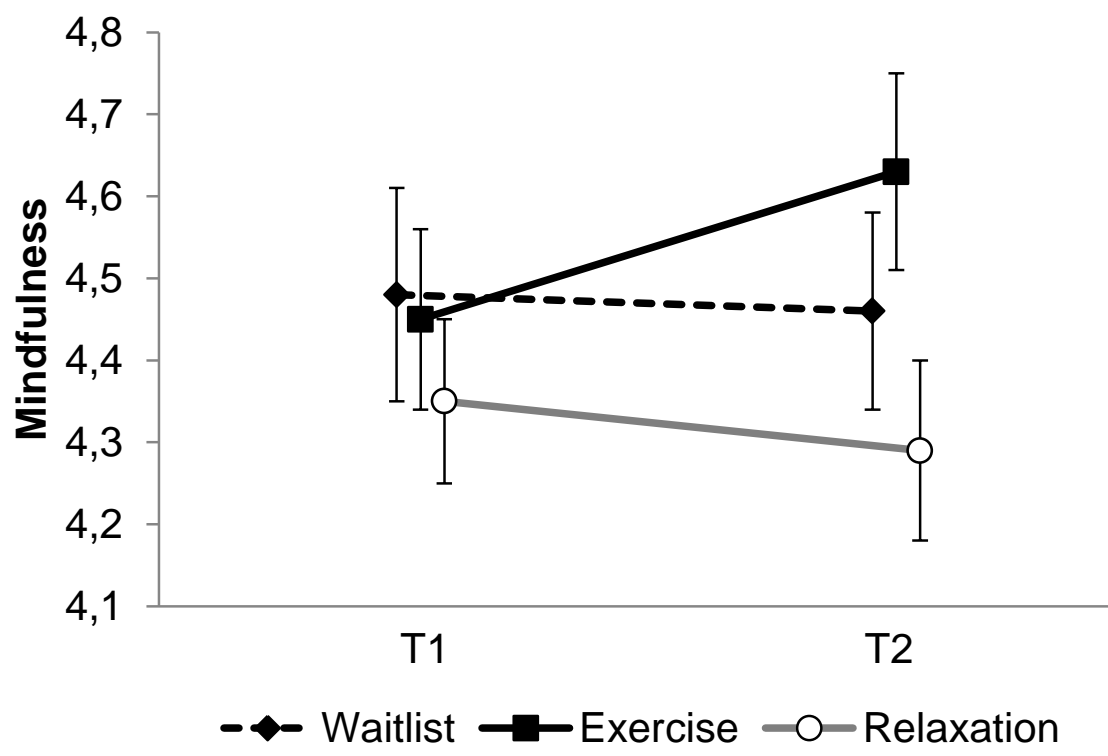

Figure 2. Mean scores of mindfulness before (T1) and after (T2) the intervention for 101 participants reporting less than 60 minutes of regular physical exercise per week at T1. Error bars represent standard errors of the means. 